\title{
A population-based cohort study of 394,401 children followed for ten years exhibits sustained effectiveness of scoliosis screening
}

Daniel Y. T. Fong, PhD, Kenneth M.C. Cheung, MD, Yat-Wa Wong, MD, Yuen-Yin Wan, MPhil, Chun-Fan Lee, PhD, Tsz-Ping Lam, FHKAM(Ortho) , Jack C.Y. Cheng, MD, Bobby K.W. Ng, MD, Keith D.K. Luk, MCH

Daniel Y. T. Fong, PhD

School of Nursing, The University of Hong Kong, 21, Sassoon Road, Hong Kong

Kenneth M. C. Cheung, MD

Department of Orthopaedics and Traumatology, The University of Hong Kong, Pokfulam, Hong Kong SAR, China

Yat-Wa Wong, MD

Department of Orthopaedics and Traumatology, The University of Hong Kong, Pokfulam, Hong Kong SAR, China

Yuen-Yin Wan, MPhil

School of Nursing, Li Ka Shing Faculty of Medicine, The University of Hong Kong, 21, Sassoon Road, Hong Kong

\section{Chun-Fan Lee, $\mathrm{PhD}$}

Department of Biostatistics of the Singapore Clinical Research Institute, and Centre for 
Quantitative Medicine of the Duke-NUS Graduate Medical School, Singapore

Tsz-Ping Lam, FHKAM (Ortho)

Department of Orthopaedics and Traumatology, The Chinese University of Hong Kong, Hong Kong SAR, China

Jack C. Y. Cheng, MD

Department of Orthopaedics and Traumatology, The Chinese University of Hong Kong, Hong Kong SAR, China

Bobby K. W. Ng, MD

Department of Orthopaedics and Traumatology, Prince of Wales Hospital, Hong Kong SAR, China

Keith D. K. Luk, MCH

Department of Orthopaedics and Traumatology, The University of Hong Kong, Pokfulam, Hong Kong SAR, China

Corresponding author: Keith D. K. Luk

Address: Department of Orthopaedics and Traumatology, The University of Hong Kong, 5/F Professorial block, Queen Mary Hospital, Pokfulam, Hong Kong.

Phone: (+852) 22554254

Fax: (+852) 28174392

E-mail: hrmoldk@hku.hk 


\section{ABSTRACT}

BACKGROUND CONTEXT: The value of scoliosis screening has been recently shown in a multicentre randomised controlled trial. However, the long-term sustainability of the clinical effectiveness of scoliosis screening as a routine health service remains unknown.

PURPOSE: To assess the sustainability of the clinical effectiveness of school scoliosis screening.

STUDY DESIGN/SETTING: A large population-based cohort study with a ten-year follow-up.

PATIENT SAMPLE: Three hundred ninety-four thousand four hundred and one students who were in fifth grade during the five academic years from 1995/1996 to 1999/2000 formed five consecutive annual cohorts. The students were eligible for the Hong Kong scoliosis screening programme, with their screening history and medical records until their nineteenth birthdays being assessed.

OUTCOME MEASURES: Development of adolescent idiopathic scoliosis by the 19 years of age and the Cobb angle.

METHODS: The clinical effectiveness of scoliosis screening was assessed by referral rate for radiographic diagnosis, sensitivity, specificity, and predictive values. Funding Source: Central Policy Unit of the Government of the Hong Kong Special Administrative Region and the Research Grants Council of the Hong Kong Special Administrative 
Region, China (Project No.: HKU 7003-PPR-09). Potential Conflict of interest-associated biases: Nothing to disclose.

RESULTS: A total of 306,144 (78\%) students participated in scoliosis screening, which used a two-tier system. The prevalence of curves $\geq 20^{\circ}$ was $1.8 \%$ (95\% confidence interval [CI] 1.7\%-1.8\%), while the referral rates for radiography, the sensitivity, and the positive predictive value (PPV) for curves $\geq 20^{\circ}$ were $4.1 \%$ (95\% CI $4.0 \%-4.2 \%$ ), $91 \%$ (95\% CI 90\%-92\%), and 40\% (95\% CI 39\%-41\%), respectively. Across the five consecutive annual cohorts, the prevalence and sensitivity for curves $\geq 20^{\circ}$ increased by 0.23\% (95\% CI 0.21\%-0.25\%; $\mathrm{p}<.001$ ) and 0.76\% (95\% CI 0.43\%-1.04\%; $\mathrm{p}<.001$ ) per year, respectively; however, the PPV was reduced by 1.71\% (95\% CI 1.09\%-2.33\%; $\mathrm{p}<.001$ ) per year.

CONCLUSIONS: This report describes the first large population-based study with a long-term follow-up indicating that a scoliosis screening programme can have sustained clinical effectiveness in identifying patients with adolescent idiopathic scoliosis needing clinical observation. As the prevalence of adolescent idiopathic scoliosis increases, scoliosis screening should be continued as a routine health service in schools or by general practitioners if there is no scoliosis screening policy.

Keywords: Adolescent idiopathic scoliosis; clinical effectiveness; scoliosis screening, sustainability 


\section{1}

2 School scoliosis screening has been used to identify individuals with adolescent

3 idiopathic scoliosis (AIS) who require clinical follow-up or conservative brace treatment,

4 with an aim of reducing the risk of requiring invasive spinal fusion surgery [1]. However,

5 despite its long history of more than 60 years, its use as a routine health service has been

6 heavily debated [2]. In 2004, the United States Preventive Services Task Force (USPSTF)

7 recommended against scoliosis screening as a routine health service, primarily because of

8 the lack of effective screening tools and inadequate evidence supporting that bracing AIS

9 patients reduces the risk of progression and hence the need for surgery [3]. This finding

10 led to the abolishment of screening programmes or reduced enthusiasm towards scoliosis

11 screening around the globe [4]. However, a recent multicentre randomised controlled trial

12 has shown that brace treatment can effectively reduce the risk of progression of AIS at

13 skeletal maturity [5]. This confirms the value of scoliosis screening for the early detection

14 of AIS [6].

15

16 The clinical effectiveness of scoliosis screening has been assessed in numerous studies of

17 different designs, which have been synthesised in a systematic review and a

18 meta-analysis [7, 8]. The systematic review covered 28 studies published between 1977

19 and 2004 and concluded that there was sufficient evidence to suggest that school scoliosis 
1 screening is safe, may detect cases of AIS at early stages, and may reduce the risk of

2 surgery [7]. In contrast, the above-mentioned meta-analysis focused on 36 retrospective

3 cohort studies that were published from 1977 to 2005 and concluded that there was

4 substantial heterogeneity across the studies, that the use of the forward bending test (FBT)

5 alone is inadequate, and that there is a need for a large retrospective cohort study with

6 sufficient follow-up [8]. To address this challenge, a large population-based retrospective

7 cohort study was later conducted that followed 157,444 students from the age of 10 until

8 they were 19 years old who were diagnosed with scoliosis via a two-tier screening

9 protocol in Hong Kong [9]. The results showed that the programme was clinically

10 effective in detecting significant curves, with a low referral rate for radiography and with

11 a cost that was close to or lower than other scoliosis screening programmes [9-11]. This

12 new evidence initiated a review of the best available evidence by an international task

13 force, which concluded that there is scientific evidence to support the technical efficacy

14 and the clinical, programme and treatment effectiveness of scoliosis screening [6].

15 However, no previous studies have assessed the sustainability of the clinical effectiveness

16 of scoliosis screening protocols. Sustained effectiveness is a critical issue and a challenge

17 for community health service initiatives [12]. A screening programme may be highly

18 effective when first implemented, but its clinical effectiveness may decrease as its

19 duration increases [13]. Inadequately designated fiscal resources, the reduced enthusiasm 
1 of the staff, unsystematic skills training, poorly articulated visions of aims and goals, and

2 a lack of support from policymakers are some of the factors that may weaken the

3 effectiveness of a community programme over time [12].

5 In Hong Kong, scoliosis screening has been conducted as a routine health service since

6 1995, thereby making it one of the regions with the longest history of routine scoliosis

7 screening in the world. Hong Kong's screening protocol was demonstrated to be

8 clinically effective for children who studied in the fifth grade during the first 2 academic

9 years after the programme was started; however, no longer-term evaluation was

10 attempted $[9,14]$. Therefore, the aim of this study is to build on our previous work to

11 assess the sustainability of the clinical effectiveness of scoliosis screening in a

12 community setting for a longer follow-up period by follow-up of the children through

13 their academic years until they reached an age of 19 years.

\section{Materials and methods}

16 This study was approved by the Institutional Review Board of the University of Hong

17 Kong/Hospital Authority Hong Kong West Cluster (UW 09-113).

18 Study design

19 We conducted a population-based cohort study of students who were in the fifth grade 
1 during the academic years 1995/1996 to 1999/2000. Medical records, which included

2 their screening history for scoliosis until they reached an age of 19 years, were retrieved.

3 Ethics approval was obtained from the local authority.

\section{School scoliosis screening programme in Hong Kong}

6 Detailed procedures of the Hong Kong scoliosis screening programme have been

7 described elsewhere [9-11]. Briefly, it is a two-tier programme. Students in tier 1 are

8 screened in regional clinics using the FBT and by measuring the angle of trunk rotation

9 (ATR) using a scoliometer. Those with an ATR $\geq 5^{\circ}$ but $<15^{\circ}$ are further screened by

10 moiré topography, and students with a difference of two or more moiré lines between the

11 left and right sides of the back or presenting with concomitant significant clinical signs of

12 spinal deformity are referred for radiographic diagnosis. Those with an ATR $\geq 15^{\circ}$ are

13 referred directly to a specialist hospital for radiographic diagnosis without undergoing

14 moiré topography. Patients diagnosed with AIS are managed and followed up in one of

15 the only two public specialist hospitals that manage scoliosis in Hong Kong. Students in

16 the fifth grade or those who have reached 10 years of age are eligible for screening. They

17 are screened biennially or more frequently if there are indications of AIS at the initial

18 screening, until they reach an age of 19 years. Children diagnosed with AIS are followed

up at a specialist hospital. 
2 The screening protocol was developed by the Department of Orthopaedics and

3 Traumatology of the University of Hong Kong. It was first implemented in 1995 and is

4 managed by the Department of Health of the Hong Kong Special Administrative Region

5 Government as a voluntary programme under an annual national health assessment

6 scheme for adolescents.

7

8 Data collection

9 Data were obtained from the Department of Health and the two specialist hospitals that

10 manage scoliosis patients. We collected data on the demographics, school grades, and

11 screening results of each visit for all fifth-grade students during the academic years

$121995 / 1996$ to 1999/2000 until they reached an age of 19 years. For the AIS patients who

13 were followed up at a specialist hospital, their referral sources, radiographic

14 measurements, body height, and treatment received (brace or surgery) at each clinical

15 visit were also recorded by trained personnel. All data were meticulously noted and

16 cross-checked with the original medical records, when necessary.

18 Statistical analysis

19 The fifth-grade students in Hong Kong in each academic year from 1995/1996 to 
1 1999/2000 formed five annual cohorts, and the clinical effectiveness of the school

2 scoliosis screening was assessed for each cohort. For each annual cohort, the referral rate,

3 sensitivity, specificity, positive predictive value (PPV), and negative predictive value

4 (NPV) for the different degrees of spinal deformity during adolescence were obtained

5 [8-10]. The referral rate was defined as the percentage of screened students referred for

6 radiography. The sensitivity and specificity were defined as the proportion of AIS patients

7 in whom AIS was detected by screening and the proportion of non-AIS subjects who

8 were negative for AIS according to the screening, respectively. The PPV was defined as

9 the proportion of referred students with AIS detected before they reached the age of 19

10 years, while NPV was defined as the proportion of non-referred students who did not

11 develop AIS during adolescence. Sensitivity and specificity are also known as accuracy

12 measures, whereas PPV and NPV are called utility measures because while the latter pair

13 can be influenced by the prevalence of AIS patients, the former pair cannot [15]. Note

14 that students who were referred for radiography but did not visit a screening clinic or a

15 specialist hospital for assessment were considered negative for AIS.

16 All estimates were accompanied by exact 95\% confidence intervals (CI) based on a

17 binomial distribution, and a 5\% level of significance was used for all significance tests.

18 The data management and analysis were performed using the Statistical Analysis System

19 (SAS) version 9.1 (SAS, NC) [16]. 


\section{Results}

3 According to national official statistics from the Education Bureau of Hong Kong,

$4 \quad 394,401$ students studied in the fifth grade during the academic years 1995/1996 to

5 1999/2000. Their disposition in the school scoliosis screening process is shown in fig 1. A

6 total of 88,257 students did not participate in the screening; of that number, scoliosis was

7 detected in $126(0.1 \%)$ before the screening, and they were therefore not screened.

8 Among those who elected not to be screened, 515 (0.6\%, 95\% CI 0.5\%-0.6\%) of them

9 were diagnosed with AIS by the time they were 19 years old. Among the 306,144 (78\%)

10 children who participated in the school scoliosis screening, 9,726 (3.2\%, 95\% CI

$113.1 \%-3.2 \%$ ) were referred for radiography by ATR or moiré topography, and 12,536

12 (4.1\%, 95\% CI 4.0\%-4.2\%) were referred when significant clinical signs, including

13 uneven shoulder height, pelvic tilt, rib or loin hump, or scapular prominence and truncal

14 shift, were detected.

16 Among the students screened, the prevalence rates of AIS for different curve magnitudes

17 and treatment statuses at 19 years of age are summarised in table 1 . The prevalence of

18 AIS among the screened students was 3.5\% (95\% CI 3.5\%-3.6\%), which was

19 significantly higher than that among students who chose not to be screened $(\mathrm{p}<.001)$. The 
1 incidence of AIS and its treatment were significantly more prevalent in girls than in boys

$2 \quad(\mathrm{p}<.001$ using Fisher's exact test), with the ratio of girls to boys increasing with a larger

3 curve size. The prevalence of curves $\geq 10^{\circ}$ by the age of 19 years in the annual cohort

4 increased linearly each year, from 2.3\% in fifth-grade students in 1995/1996 to 4.7\% in

5 those in the 1999/2000 cohort, i.e., an average of 0.61\% per year (95\% CI 0.58\%-0.64\%;

$6 \mathrm{p}<.001$ using Binomial regression) (Fig. 2). Similarly, the prevalence of curves $\geq 20^{\circ}$

7 increased from $1.3 \%$ for the fifth-grade students in $1995 / 96$ to $2.2 \%$ for those in the

8 1999/2000 cohort, i.e., an average of 0.23\% per year (95\% CI 0.21\%-0.25\%; $<<.001$ ).

9 This increase in prevalence was accompanied by an increase in the referral rate for

10 radiography from $2.7 \%$ (95\% CI $2.5 \%-2.8 \%$ ) in the $1995 / 1996$ cohort to $5.5 \%$ (95\% CI

$115.3 \%-5.7 \%$ ) in the 1999/2000 cohort. Coincidentally, there was also an annual increase

12 of $2 \%$ in the participation rate, which reached $81 \%$ of fifth-grade students in 1999/2000.

14 Table 2 presents the results for the clinical effectiveness of school scoliosis screening,

15 and figs 3 and 4 depict the longitudinal changes over the five annual cohorts. For

16 detecting curves $\geq 10^{\circ}$, the four measures of clinical effectiveness were generally stable,

17 with a slight increase in the sensitivity of $0.76 \%$ per year (95\% CI $0.43 \%-1.04 \%$;

$18 \mathrm{p}<.001$ ), resulting in an increase from $92 \%$ in the $1995 / 1996$ cohort to $95 \%$ in the

19 1999/2000 cohort. Moreover, both the specificity and NPV remained consistently above 
$199 \%$. For detecting curves $\geq 20^{\circ}$, the sensitivity increased by $0.87 \%$ per year ( $95 \%$ CI

2 0.33\%-1.40\%; $\mathrm{p}=.001$ ), from $89 \%$ in the $1995 / 1996$ cohort to $91 \%$ in the $1999 / 2000$

3 cohort, despite an annual reduction of $0.5 \%$ in specificity and a $1.71 \%$ (95\% CI

$4 \quad 1.09 \%-2.33 \% ; \mathrm{p}<.001$ ) reduction in the PPV. Nevertheless, the specificity remained

5 above $96 \%$ and the PPV remained above $37 \%$. The NPV was consistently above 99\%.

7 Discussion

8 To our knowledge, this is the first large population-based representative study to assess

9 the sustainability of the clinical effectiveness of school scoliosis screening as a routine

10 health service in the community. The prevalence of AIS in Hong Kong has been

11 increasing since the screening programme began, but the sensitivity and predictive value

12 of the Hong Kong screening programme for detecting AIS were sustained over five

13 consecutive annual cohorts of students who were followed for at least 10 years.

14 A screening programme should preferably be highly accurate, with the sensitivity,

15 specificity, PPV, and NPV as high as possible. However, the implementation of a

16 screening programme will inevitably have suboptimal accuracy due to behavioural

17 noncompliance. It has been recommended that the sensitivity and specificity of a

18 screening programme should be $70 \%$ or more, while the PPV is often between $30 \%$ and

$1950 \%$ [17]. To detect curves $\geq 20^{\circ}$, a degree that merits at least clinical monitoring, the 
1 sensitivity and specificity of the Hong Kong scoliosis screening protocol were $91 \%$ and

$298 \%$, respectively, which are far above the recommended standard of $70 \%$. Moreover, the

3 corresponding NPV was almost $100 \%$ and the PPV was $40 \%$, both of which fall within

4 the commonly reported range. Hence, the Hong Kong scoliosis screening programme has

5 demonstrated acceptable accuracy in detecting curves deserving of clinical monitoring.

6 The PPV for detecting curves $\geq 20^{\circ}$ in the Hong Kong scoliosis screening programme

7 decreased over the annual cohorts. This is unlikely to be due to the influence of the

8 corresponding prevalence, as the prevalence rate of curves $\geq 20^{\circ}$ increased over the

9 annual cohorts, which should have increased the PPV [15]. The reduction in the PPV for

10 curves $\geq 20^{\circ}$ may be a consequence of an increase in the referral rates for radiography.

11 Given that the PPV for curves $\geq 10^{\circ}$ was maintained at a high level and the sensitivity for

12 detecting curves $\geq 10^{\circ}$ or $\geq 20^{\circ}$ has increased, the additional referral rate is unlikely to be

13 a result of a decline in screening skills, but it may be due to increased concerns about the

14 disorder as more AIS patients were identified.

16 The screening protocol adopted in Hong Kong continued to be more effective than other

17 existing programs even several years after its implementation in 1995 [8]. Starting a

18 school scoliosis-screening programme as an additional health service in the community

19 requires investing enormous efforts not only in developing the screening protocol but also 
1 in setting up procedures across various units in the healthcare system, obtaining screening

2 equipment, and training staff. The skill of the staff is perhaps the most highly variable

3 factor that influences the clinical effectiveness of scoliosis screening programmes. The

4 personnel involved in scoliosis screening may be meticulous and attentive when they

5 have first learned their screening skills, but this skill may not be sustained over years of

6 routine administration and staff rotation. Staff enthusiasm is another important factor for

7 sustaining the clinical effectiveness of screening. The recommendation against scoliosis

8 screening made by the United States Preventive Services Task Force reduced the

9 enthusiasm for scoliosis screening [3]. This recommendation even led to the cessation of

10 scoliosis screening by nursing associations [18]. Sustaining the clinical effectiveness of a

11 screening programme is essential to ensure the long-term feasibility and value of

12 screening. The success of the Hong Kong scoliosis-screening programme in maintaining

13 an effective performance is most likely because the programme was highly centralised

14 and was coordinated by the Department of Health with support and supervision from a

15 University Department. This ensured strict adherence to the screening protocol, and

16 screening personnel were properly trained before they could begin screening. The

17 Department of Orthopaedics and Traumatology of The University of Hong Kong had

18 been conducting regular courses for screening doctors to ensure their skills were

19 maintained and to train new screeners. All screening personnel were also monitored for a 
1 month after training before they could screen students independently.

3 Participation in the Hong Kong screening programme has also been improving, from the

4 previously reported $73 \%$ among the first two annual cohorts of students to $78 \%$ when the

5 next three annual cohorts were also considered. This participation rate was higher than

6 those reported in Singapore and Minnesota and is comparable to the highest reported

7 participation rate in the literature of approximately $80 \%$ in the Netherlands [19-21].

8 Indeed, since the implementation of the Hong Kong scoliosis-screening program, there

9 have been regular radio and television programmes to educate the public about the

10 importance of scoliosis screening. The increase in the participation rate could be due to

11 the public's increased awareness of scoliosis after the programme was implemented.

12 The estimated prevalence rates of AIS with curves $\geq 10^{\circ}$ and $\geq 20^{\circ}$ by the age of 19

13 among students in Hong Kong have gradually increased over each annual cohort. There

14 are several reasons for this increase. First, the scoliosis-screening programme in Hong

15 Kong is voluntary, and the increased participation rate may reflect that there are more

16 children who are concerned about spinal deformity being screened. Second, the increased

17 screening sensitivity indicates that the chances of identifying an AIS patient through

18 screening have increased. In other words, we may have approached the actual prevalence

19 only in the later annual cohorts. Third, the increased urbanisation in Hong Kong may also 
1 have contributed to the increased prevalence of AIS. Indeed, there was also an increase in

2 the annual prevalence of AIS among children screened in Korea, from $1.7 \%$ in 2000 to

$36.2 \%$ in 2008 [22]. An earlier study in Japan reported that urbanised regions had a higher

4 incidence of AIS than rural regions [23]. The average annual growth in the percentage of

5 the total population living in urban areas in Korea between 2000 and 2010 was 1.22,

6 whereas the equivalent figure for Hong Kong between 1995 and 2000 was even higher, at

$7 \quad 2.14 \%$, which was slightly above the global figure of 2.11\% [24]. Although the

8 association between urbanisation and the development of AIS is not fully understood,

9 urbanisation may cause children to mature earlier due to improved nutritional standards

$10[25,26]$. Because children who mature early have been shown to have a significantly

11 longer pubescent period and increased height gain during puberty,[27] the risk of curve

12 progression may be high.

14 Compared with our previous results based on the first two annual cohorts, the ratio of

15 girls to boys has decreased from 2.7 to 2.2 for curves $\geq 10^{\circ}$, from 4.5 to 3.6 for curves $\geq$

$1620^{\circ}$, from 8.1 to 5.5 for curves $\geq 40^{\circ}$, and from 8.4 to 5.3 for those receiving treatment.

17 Although the prevalence of AIS has certainly increased among girls, the probability of

18 AIS development among boys has been gradually increasing. This finding implies that

19 the previous suggestion of the selective screening of girls only may not be appropriate, at 
1 least in the Hong Kong setting [28-30].

2

3 Whether students should be screened for AIS depends on not only the availability of a

4 clinically effective screening program but also on the availability of an efficacious

5 conservative treatment [31]. For a very long time, bracing has been the most commonly

6 used option for the treatment of AIS, but strong evidence to support its efficacy in

7 controlling curve progression has been reported only recently [5, 32]. A study that was

8 started as a randomised controlled trial and later incorporated a preference cohort

9 reported that $72 \%$ of braced patients had curves below $50^{\circ}$ at skeletal maturity, which

10 was significantly higher than the corresponding $48 \%$ of patients who were not braced

11 (adjusted odds ratio $=4.1$ ). Another clinical trial of a similar design but with both the

12 randomised and preference cohorts planned as concurrent components has had its

13 feasibility recently assessed and confirmed in the local setting in Hong Kong [33]. The

14 strong evidence and continual efforts in support of the efficacy of bracing have strengthened the need for screening for idiopathic scoliosis so that bracing can be applied early to prevent curve progression and hence reduce the risk of surgery.

18 We have not examined the cost of scoliosis screening because it has been previously

19 reported [11]. In Hong Kong, if at least 52\% of the braced students would have required 
1 surgery had they not been braced, the cost saved from preventing the need for surgery

2 could offset the costs of screening and follow-up. In contrast, $65 \%$ or more would be

3 needed in Singapore to break even between the costs of surgery and the costs of screening

4 and follow-up [34]. In general, it is difficult to compare costs associated with screening

5 across countries because of the differences in their health-care systems and economies.

6 Nevertheless, the costs of screening in Hong Kong appeared to be comparable with those

7 in the U.S.

8

9 Conclusions

10 Sustaining the clinical effectiveness of a scoliosis screening programme requires not only

11 an effective screening protocol but also concerted effort from administrators, clinicians,

12 and all frontline staff involved in the screening. Regular training and monitoring of

13 screening personnel to ensure the optimal delivery of screening procedures and publicity

14 to ensure high participation rates in scoliosis screening play vital roles in sustaining the

15 clinical effectiveness of the Hong Kong scoliosis screening programme. Despite the

16 controversy surrounding scoliosis screening, this screening has provided information

17 essential to the understanding of the epidemiology of AIS [35]. The increase in the

18 prevalence of AIS in Hong Kong, together with the consistently clinically effective

19 scoliosis screening protocol, supports the continuation of scoliosis screening in schools or 
1 by general practitioners in places without a scoliosis screening policy.

2 


\section{REFERENCES}

2 1. Richards BS, Vitale MG. Screening for idiopathic scoliosis in adolescents. An information statement. 2008;90(1):195-8.

$4 \quad 2 . \quad$ Fong DYT, Luk KDK. Should screening for scoliosis be conducted? 2012;24(1-2):46-49.

6 3. USPSTF, Screening for idiopathic scoliosis in adolescents: recommendation statement. Rockville, MD: Agency for Healthcare Research and Quality; 2004.

8 4. Adobor RD, Riise RB, Sorensen R, Kibsgard TJ, Steen H, Brox JI. Scoliosis detection, patient characteristics, referral patterns and treatment in the absence of a screening program in Norway. Scoliosis 2012;7(1):18.

5. Weinstein SL, Dolan LA, Wright JG, Dobbs MB. Effects of Bracing in Adolescents with Idiopathic Scoliosis. N Engl J Med 2013.

6. Labelle H, Richards SB, De Kleuver M, et al. Screening for adolescent idiopathic scoliosis: an information statement by the scoliosis research society international task force. Scoliosis 2013;31(8(1)):17.

7. Sabirin J, Bakri R, Buang SN, Abdullah AT, Shapie A. School scoliosis screening programme-a systematic review. The Medical journal of Malaysia 2010;65(4):261-7. 
of school scoliosis screening. Spine 2010;35(10):1061-71.

2 9. Luk KD, Lee CF, Cheung KM, et al. Clinical effectiveness of school screening for adolescent idiopathic scoliosis: a large population-based retrospective cohort study. Spine 2010;35(17):1607-14.

10. Lee CF, Fong DY, Cheung KM, et al. Referral criteria for school scoliosis screening: assessment and recommendations based on a large longitudinally followed cohort. Spine 2010;35(25):E1492-8.

8 11. Lee CF, Fong DY, Cheung KM, et al. Costs of school scoliosis screening: a large, population-based study. Spine 2010;35(26):2266-72.

12. The Finance Project, Sustaining Comprehensive Community Initiatives: Key Elements for Success, 2002, The Finance Project: Washington, DC. p. 20.

13. Lawless S. Sustaining chlamydia screening is difficult. Journal of primary health care 2010;2(4):347.

14. Yawn BP, Yawn RA, Hodge D, et al. A population-based study of school scoliosis screening. 1999;282(15):1427-32.

15. Zhou X-h, McClish DK, Obuchowski NA, Statistical methods in diagnostic medicine. Hoboken, N.J.: Wiley; 2011.

16. SAS Institute Inc, SAS/STAT user's guide, version 8. Cary, N.C: SAS Institute Inc; 1999. 
1 17. Glascoe FP Standards for Screening Test Construction. 2005.

2 18. West Virginia Council of School Nurses, Recommendation for postural screenings, 2006, West Virginia Council of School Nurses.

4 19. Bunge EM, de Koning HJ, brace trial group. Bracing patients with idiopathic scoliosis: design of the Dutch randomized controlled treatment trial. 2008;9(57).

62 20. Lonstein JE, Bjorklund S, Wanninger MH, Nelson RP. Voluntary school screening for scoliosis in Minnesota. 1982;64(4):481-488.

8 21. Wong HK, Hui JHP, Rajan U, Chia HP. Idiopathic scoliosis in Singapore schoolchildren. A prevalence study 15 years into the screening program. 2005;30(10):1188-1196.

11 22. Suh SW, Modi HN, Yang JH, Hong JY. Idiopathic scoliosis in Korean schoolchildren: a prospective screening study of over 1 million children. European Spinal Deformity Society, and the European Section of the Cervical Spine Research Society 2011;20(7):1087-94.

23. Ohtsuka Y, Yamagata M, Arai S, Kitahara H, Minami S. School screening for scoliosis by the Chiba University Medical School screening program. Results of 1.24 million students over an 8-year period. 1988;13(11):1251-7. 
Affairs, Editor 2001, United Nations: United States of America.

2 25. Pasquet P, Biyong AMD, Rikong-Adie H, Befidi-Mengue R, Garba MT, Froment

A. Age at menarche and urbanization in Cameroon: current status and secular trends. Ann Hum Biol 1999;26(1):89-97.

26. Tanner JM, Foetus into man : physical growth from conception to maturity. Cambridge, Mass.: Harvard University Press; 1990.

27. Pantsiotou S, Papadimitriou A, Douros K, Priftis K, Nicolaidou P, Fretzayas A. Maturational tempo differences in relation to the timing of the onset of puberty in girls. 2008;97(2):217-20.

28. Goldberg CJ, Dowling FE, Fogarty EE, Moore DP. School scoliosis screening and the United States Preventive Services Task Force. An examination of long-term results. Spine 1995;20(12):1368-74.

29. Hansen TB. [Adolescent idiopathic scoliosis among girls in the Herning region. A follow-up of girls with adolescent idiopathic scoliosis found in an earlier screening at school]. Ugeskrift for laeger 1994;156(35):4979-82.

30. Al-Turaiki MH, Al-Falahi LA, Saad Eddin M, Gremly M. School screening for scoliosis in Riyadh. 1994;15(4):277-280.

31. Lonstein JE. Adolescent idiopathic scoliosis. 1994;344(8934):1407-1412.

32. Maruyama T, Grivas TB, Kaspiris A. Effectiveness and outcomes of brace 
treatment: a systematic review. Physiother Theory Pract 2011;27(1):26-42.

2 33. Fong DYT, Cheung KMC, Wong Y, et al, Is there a better alternative to a randomized control design for assessing the efficacy and effectiveness of bracing in AIS?, in 46th Annual Meeting Scoliosis Research Society2011: Louisville.

5 34. Thilagaratnam S. School-based screening for scoliosis: is it cost-effective? Singapore Med J 2007;48(11):1012-7.

7 35. Grivas TB, Vasiliadis E, Savvidou OD, Triantafyllopoulos G. What a school screening program could contribute in clinical research of idiopathic scoliosis aetiology. 2008;30(10):752-762. 


\section{FIGURE LEGENDS}

Fig 1 School screening for adolescent idiopathic scoliosis (AIS)

Fig 2 Prevalence of patients with adolescent idiopathic scoliosis

Fig 3 Clinical effectiveness of school scoliosis screening for detecting curves of at least $10^{\circ}$

Fig 4 Clinical effectiveness of school scoliosis screening for detecting curves of at least $20^{\circ}$ 


\section{EVIDENCE AND METHODS}

\section{Context}

School scoliosis screening is safe and may detect adolescent idiopathic scoliosis. Bracing is efficacious in controlling curve progression in patients with adolescent idiopathic scoliosis.

\section{Contribution}

A carefully designed and conducted scoliosis screening programme can have sustained clinical effectiveness in identifying patients with adolescent idiopathic scoliosis who require clinical monitoring or treatment

\section{Implications}

Screening for adolescent idiopathic scoliosis should be continued as a national health service in schools or by general practitioners in places without a scoliosis screening policy 
Table 1

Prevalence of Adolescent Idiopathic Scoliosis by the Age of 19 years

\begin{tabular}{lcccc}
\hline & \multicolumn{3}{c}{ Prevalence (Exact 95\% CI) } & \\
\cline { 2 - 4 } & Total & Boys & Girls & Girls:Boys \\
\hline Curves $\geq 10^{\circ}$ & $3.5 \%(3.5 \%, 3.6 \%)$ & $2.2 \%(2.1 \%, 2.2 \%)$ & $4.8 \%(4.7 \%, 5.0 \%)$ & 2.2 \\
Curves $\geq 20^{\circ}$ & $1.8 \%(1.7 \%, 1.8 \%)$ & $0.7 \%(0.7 \%, 0.8 \%)$ & $2.8 \%(2.7 \%, 2.8 \%)$ & 3.6 \\
Curves $\geq 40^{\circ}$ & $0.2 \%(0.2 \%, 0.3 \%)$ & $0.07 \%(0.06 \%, 0.09 \%)$ & $0.4 \%(0.4 \%, 0.4 \%)$ & 5.5 \\
Treatment & $0.4 \%(0.4 \%, 0.4 \%)$ & $0.1 \%(0.1 \%, 0.1 \%)$ & $0.7 \%(0.6 \%, 0.7 \%)$ & 5.3 \\
\hline
\end{tabular}


Table 2

Clinical Effectiveness of School Scoliosis Screening

\begin{tabular}{lcccc}
\hline & $\begin{array}{c}\text { Positive predictive value } \\
\text { (Exact 95\% CI) }\end{array}$ & $\begin{array}{c}\text { Negative predictive value } \\
\text { (Exact 95\% CI) }\end{array}$ & $\begin{array}{c}\text { Sensitivity } \\
\text { (Exact 95\% CI) }\end{array}$ & $\begin{array}{c}\text { Specificity } \\
\text { (Exact 95\% CI) }\end{array}$ \\
\hline Curves $\geq 10^{\circ}$ & $81.0 \%(80.3 \%, 81.7 \%)$ & $99.8 \%(99.8 \%, 99.8 \%)$ & $93.8 \%(93.3 \%, 94.3 \%)$ & $99.2 \%(99.2 \%, 99.2 \%)$ \\
Curves $\geq 20^{\circ}$ & $39.8 \%(38.9 \%, 40.6 \%)$ & $99.8 \%(99.8 \%, 99.8 \%)$ & $91.0 \%(90.2 \%, 91.7 \%)$ & $97.5 \%(97.4 \%, 97.5 \%$ \\
Curves $\geq 40^{\circ}$ & $4.6 \%(4.2 \%, 5.0 \%)$ & $99.9 \%(99.9 \%, 100 \%)$ & $77.6 \%(74.5 \%, 80.6 \%)$ & $96.1 \%(96.0 \%, 96.2 \%)$ \\
Treatment & $8.4 \%(7.9 \%, 8.9 \%)$ & $99.9 \%(99.9 \%, 99.9 \%)$ & $83.3 \%(81.1 \%, 85.3 \%)$ & $96.2 \%(96.2 \%, 96.3 \%)$ \\
\hline
\end{tabular}


Fig. 1. School screening for adolescent idiopathic scoliosis (AIS)

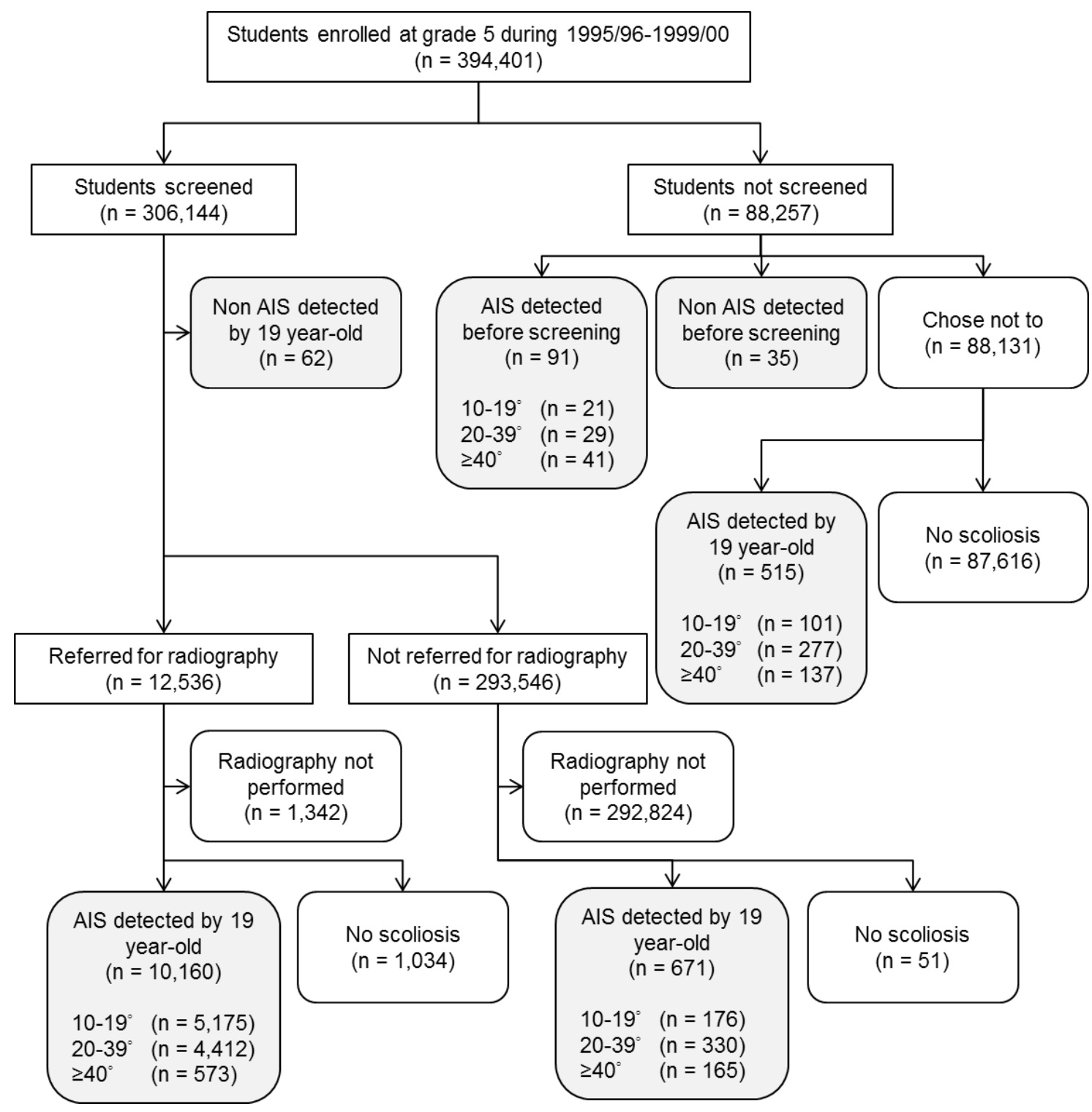


Fig. 2. Prevalence of patients with adolescent idiopathic scoliosis

(a) Cobb angle $\geq 10^{\circ}$

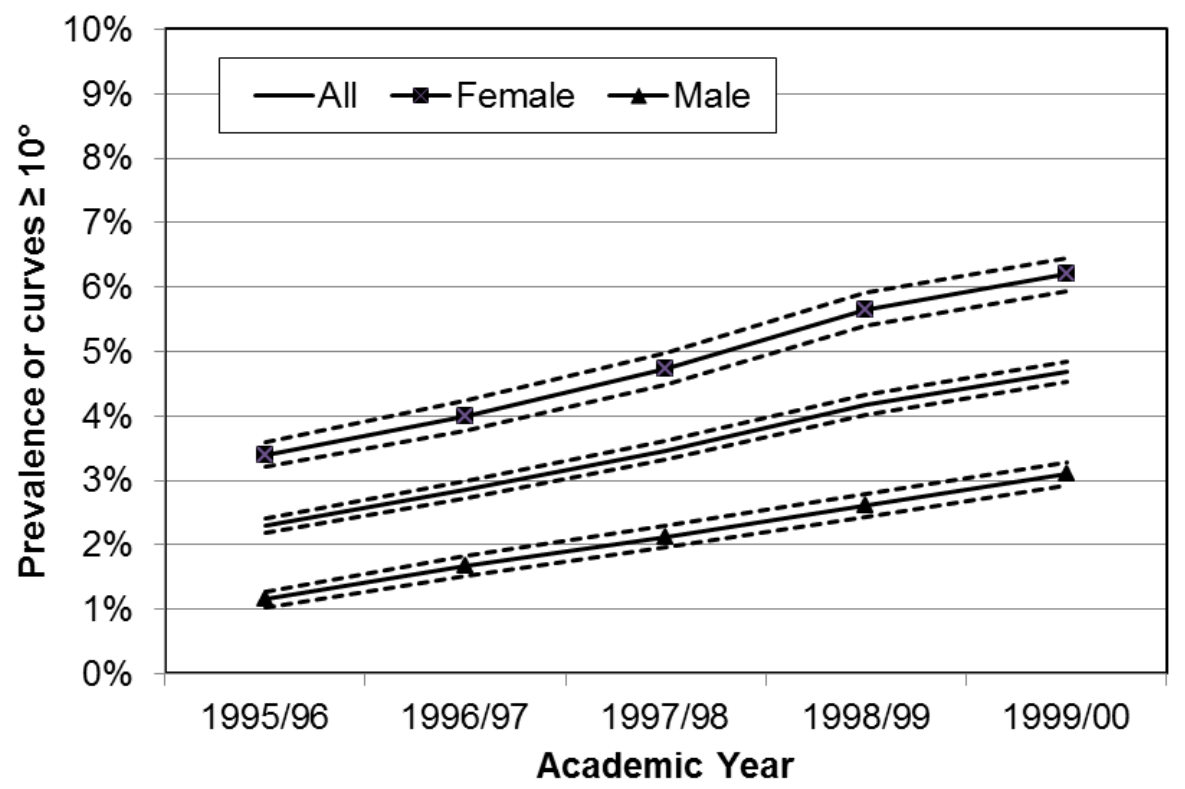

(b) Cobb angle $\geq 20^{\circ}$

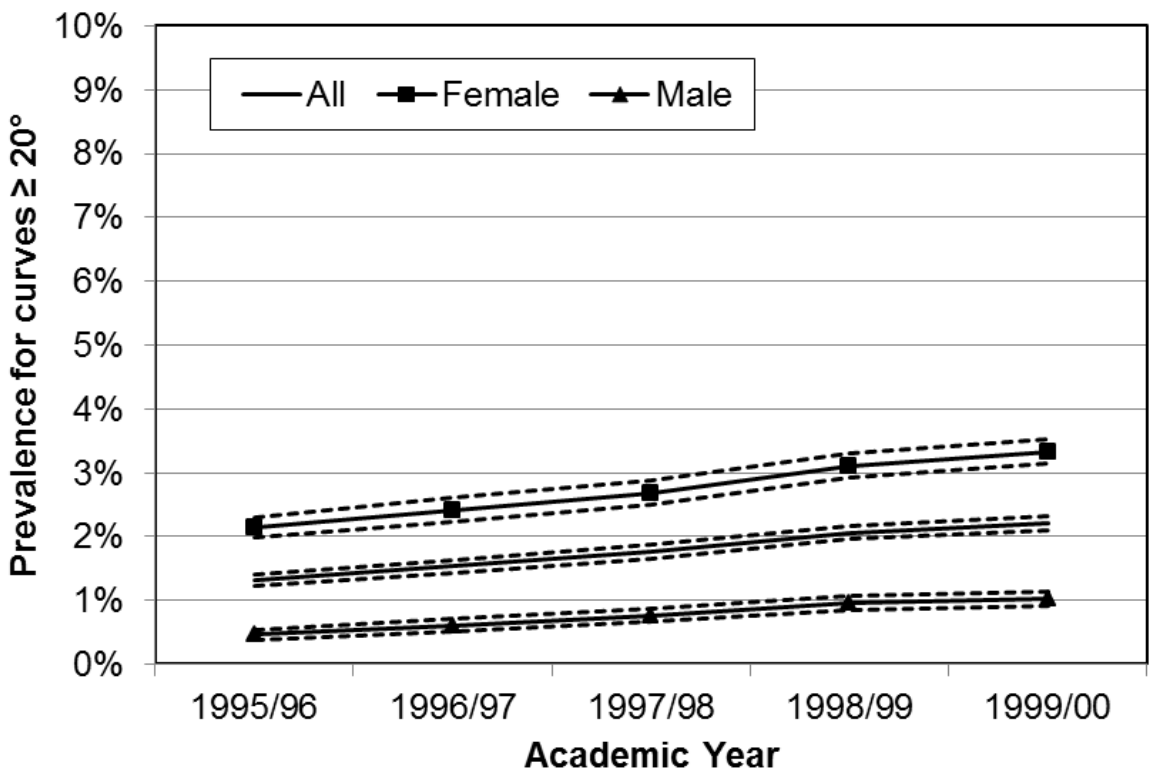


Fig. 3. Clinical effectiveness of school scoliosis screening for detecting curves of at least $10^{\circ}$

(a) Sensitivity

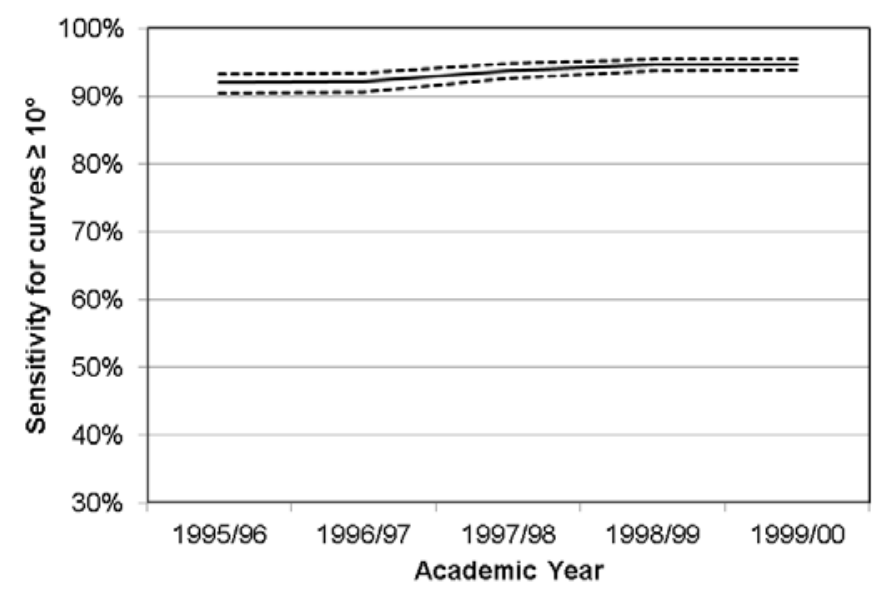

(c) Positive predictive value

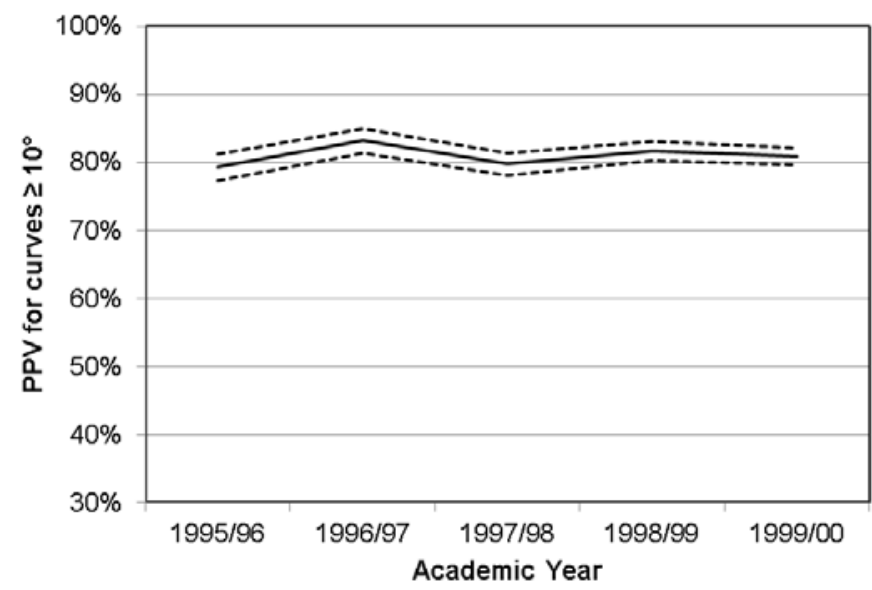

(b) Specificity

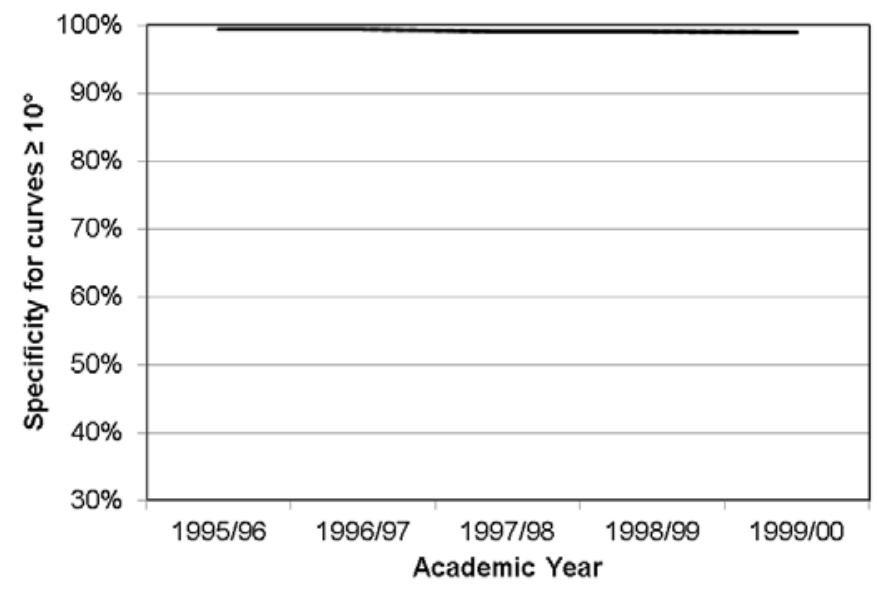

(d) Negative predictive value

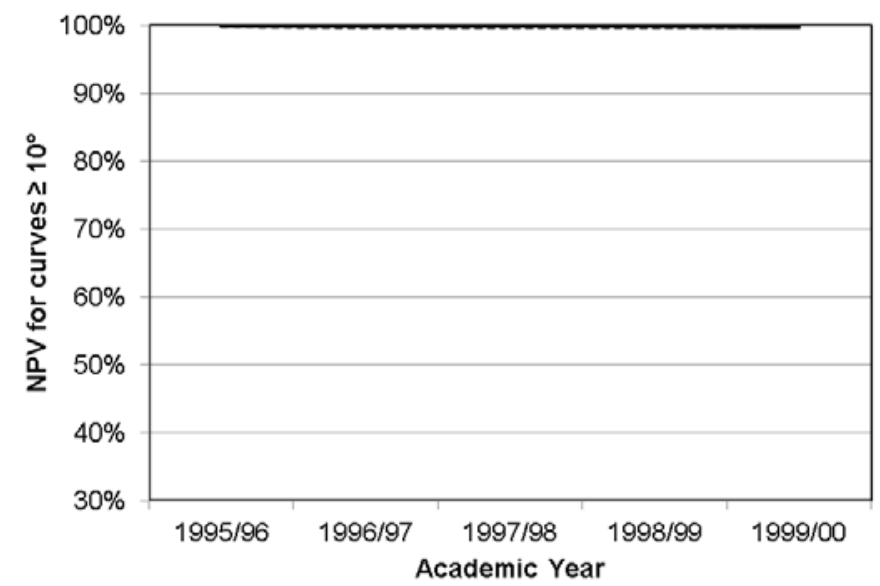


Fig. 4. Clinical effectiveness of school scoliosis screening for detecting curves of at least $20^{\circ}$

(a) Sensitivity

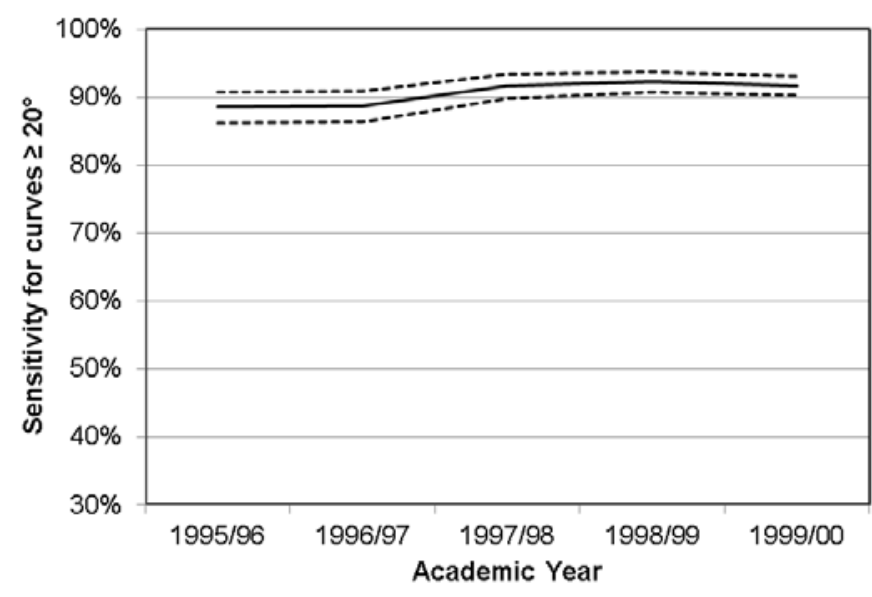

(c) Positive predictive value

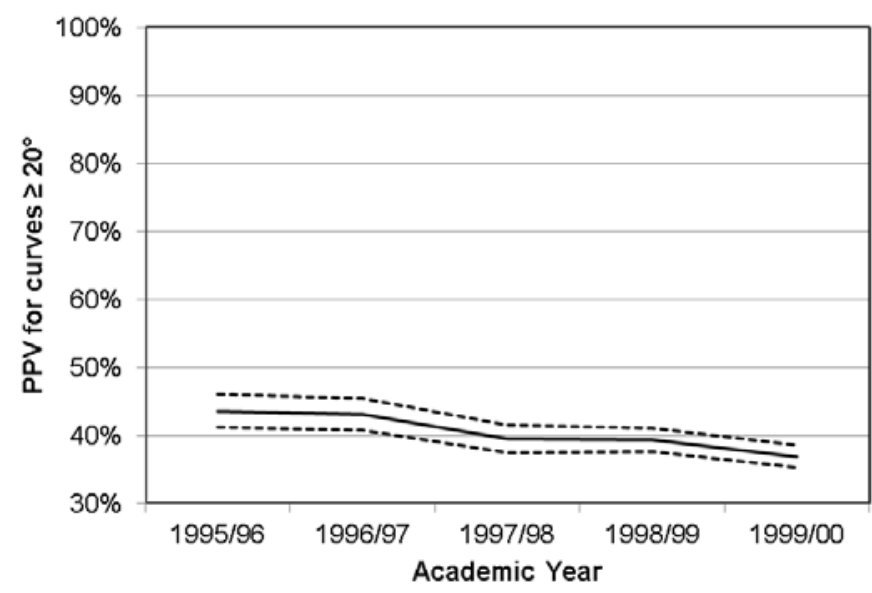

(b) Specificity

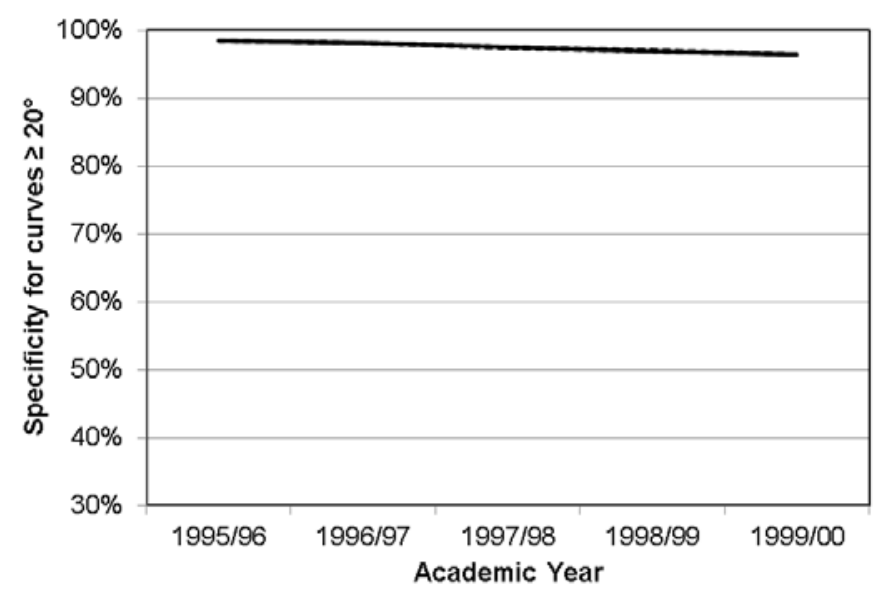

(d) Negative predictive value

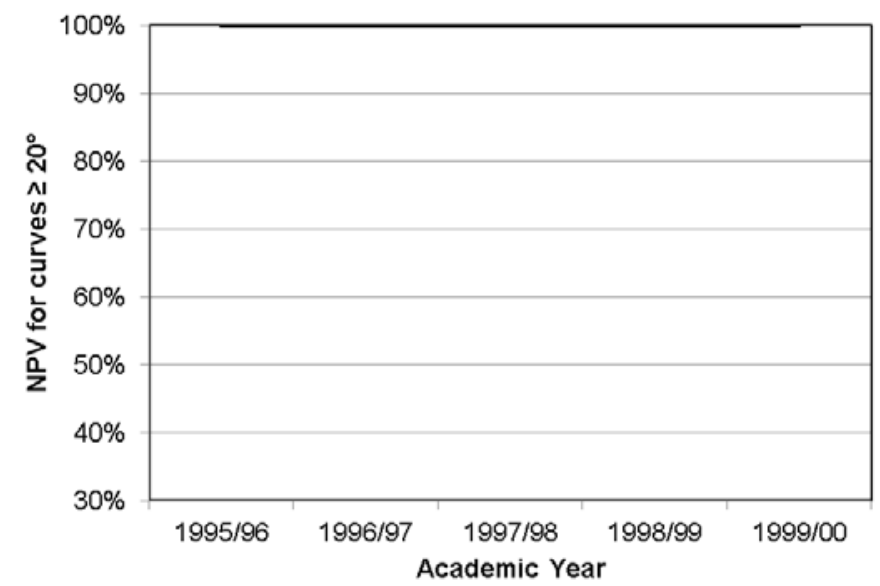

